\title{
Sur les différentes Moyennes des Grandeurs moléculaires mesurables sur un Echantillon polydispersé
}

\author{
Ö. BRYDE et A. BANDERET \\ Papirindustriens Forskningsinstitutt, Sköyen, Nort’̀̀ge, \\ et \\ Ecole Supérieure de Chimie, Mulhouse, France
}

$\mathrm{O}^{n}$ n sait que dans l'état actuel de nos techniques de préparation et de purification, les produits macromoléculaires que nous pouvons obtenir sont presque toujours constitués de mélanges contenant des molécules de masses différentes. Il est vrai qu'au prix de précautions convenables, nous savons préparer à partir de produits naturels certaines protéines qui se révèlent homogènes quand on les soumet aux critères expérimentaux les plus sensibles. Mais, même si toutes les molécules de tels échantillons ont bien la même masse, elles peuvent prendre en général toute une série de formes.

Les grandeurs qui caractérisent les macromolécules ne peuvent donc en général pas être exprimées par un seul chiffre. Il faudrait pouvoir donner la courbe de distribution de la grandeur considérée entre les molécules. Trop souvent, il faut se contenter d'une certaine valeur moyenne.

\section{$\S 1$}

Certaines valeurs moyennes sont fournies directement par l'expérience. On peut remarquer que si ces moyennes sont, formellement, de types assez variés, elles peuvent pourtant s'obtenir toutes d'après le même schéma.

Sur le mélange à étudier, on mesure une grandeur $A$ que l'on sait être la somme des grandeurs correspondantes $A_{i}$ attachées à chacune des espèces de molécules $(i)$ présentes dans le mélange.

$$
A=\Sigma A_{i}
$$

Cette grandeur $A_{\imath}$ est fonction d'une grandeur moléculaire particulière $\lambda_{i}$, d'une série d'autres grandeurs moléculaires $\mid \xi_{i} /$ et d'un certain nombre de paramètres extérieurs $|x|$ 


$$
A_{i}=A\left(\lambda_{i},\left|\xi_{i} /,\right| x /\right)
$$

Par ailleurs, il faut que la fonction $A$ puisse se mettre sous la forme

$$
A\left(\lambda_{i},\left|\xi_{i} /,\right| x /\right)=\varphi\left(\lambda_{i}\right) \cdot \psi\left(\lambda_{i},\left|\xi_{i} /,\right| x /\right)
$$

Finalement, il faut que la fonction $\psi$, tout comme la fonction A soit une fonction additive, c'est à dire que

$$
\psi=\Sigma \psi_{i}
$$

soit bien l'expression d'une réalité physique. Si toutes les trois conditions (1), (2) et (3) sont remplies, on peut calculer la valeur moyenne de $\bar{\lambda}$ que fournit la méthode de mesure envisagée.

$$
\begin{gathered}
\sum \varphi_{i} \cdot \psi_{i}=\bar{\varphi} \Sigma \psi_{i} \\
\bar{\lambda}=\bar{\varphi}^{-1}\left(\frac{\sum A\left(\lambda_{i}, / \xi_{i} /, \mid x /\right)}{\sum \psi\left(\lambda_{i}, / \xi_{i} /, \mid x /\right)}\right)
\end{gathered}
$$

$\varphi^{-1}$ représentant la fonction inverse de la fonction $\varphi$.

Voici quelques exemples tirés de la littérature. Nous avons conservé les notations originales et placé un tableau des symboles utilisés à la fin du présent article.

Tableau 1.

$$
\begin{aligned}
& \begin{array}{lllll}
\cdot \mathbf{A} & \cdot \lambda \cdot & \cdot p \cdot & \cdot \psi \cdot & \text { Référence }
\end{array} \\
& \begin{array}{cllcl}
\pi & M & M^{-1} & R T c & \\
\eta-\eta_{0} & M & M^{\alpha} & K_{m} c \eta_{0} & \\
\frac{\mathrm{d} c}{\mathrm{~d} x} & M & M & 2 A x c & \text { Lansing \& Kraemer }{ }^{1} \text {. }
\end{array} \\
& \begin{array}{ccccl}
\frac{\mathrm{d} Z}{\mathrm{~d} x}-\frac{Z}{x} & M & M & 4 A^{2} x^{2} M c & \text { Ibid. } \\
m_{n+2} & D & D & m_{n} & \text { Gralén }{ }^{2} . \\
n_{e}-n_{0} & a & a^{3} & \frac{32 \pi^{2}\left(g_{1}-g_{2}\right) G \eta c}{45 n k T\left(2 \ln \left(\frac{2 a}{b}\right)-1\right)} & \text { Wales }{ }^{3} . \\
\end{array}
\end{aligned}
$$

$\S 2$

Dans quelques cas, on dispose d'une série de valeurs moyennes pour les différentes fractions en lesquelles on a pu diviser un échantillon à étudier. 
On peut alors calculer la moyenne de ces moyennes d'après le même schéma, à cette différence près toutefois que l'additivité des fonctions $\mathrm{A}$ et $\boldsymbol{a}$ ne doit plus nécessairement répondre à une réalité physique. Voici quelques exemples.

\begin{tabular}{ccccl}
\multicolumn{1}{c}{$\mathrm{A}$} & $\cdot \lambda \cdot$ & $\cdot p \cdot$ & $\cdot \psi \cdot$ & Référence \\
$M_{z x} \cdot Z \cdot \mathrm{d} x$ & $M$ & $M$ & $Z \cdot \mathrm{d} x$ & Lansing \& Kraemer ${ }^{1}$. \\
$M_{z t z} \cdot c \cdot x \cdot \mathrm{d} x$ & $M$ & $M$ & $c \cdot x \cdot \mathrm{d} x$ & Ibid.
\end{tabular}

Jullander ${ }^{5}$ donne une méthode permettant d'obtenir graphiquement la courbe

$$
\frac{\mathrm{d} c}{\mathrm{~d} s}=f(s)
$$

que l'ont peut intégrer graphiquement. A partir de la courbe intégrale, on peut calculer une série de valeurs moyennes d'une façon purement formelle. Par analogie avec les moyennes $M_{n}, M_{w}$ et $M_{z}$ introduites par Lansing et Kraemer ${ }^{1}$ et entrées depuis dans la pratique on peut écriı

$$
\begin{aligned}
& s_{n}=\frac{\sum \frac{c_{i} s_{i}}{M_{i}}}{\sum \frac{c_{i}}{M_{i}}} \\
& s_{i c}=\frac{\sum c_{i} s_{i}}{\sum c_{i}} \\
& s_{z}=\frac{\sum c_{i} s_{i} M_{i}}{\sum c_{i} M_{i}}
\end{aligned}
$$

par analogie avec, par exemple

$$
M_{z}=\frac{\sum M_{i} c_{i} M_{i}}{\sum c_{i} M_{i}}
$$

ce choix étant justifié par le fait que $\frac{c}{M}$ représente le nombre de molécules présentes. Mais on peut aussi bien écrire, en analogie formelle

$$
s_{n}^{\prime}=\frac{\sum c_{i}}{\sum \frac{c_{i}}{s_{i}}}
$$




$$
\begin{aligned}
s_{i v} & =\frac{\sum c_{i} s_{i}}{\sum c_{i}} \\
s_{i}^{\prime} & =\frac{\sum c_{i} s_{i}^{2}}{\sum c_{i} s_{i}} .
\end{aligned}
$$

comme aussi nous pouvons écrire avec Gralén ${ }^{6}$

$$
D_{n}=\frac{\sum c_{i}}{\sum \frac{c_{i}}{D_{i}}}
$$

On se rend compte ainsi que la désignation de ce genre de moyennes par un seul indice est insuffisante *. Du reste le nombre de ces moyennes va en augmentant à mesure que se développe la chimie macromoléculaire (voir Wales ${ }^{3}, 9$ ). C'est pourquoi nous avons essayé de trouver une nomenclature systématique pour ces grandeuis.

\section{$\S \mathbf{3}$}

Observons que les fonctions $A$ et $\psi$ qui apparaissent dans la formule (4) prennent une forme plus simple par suite de l'élimination des paramètres $x$. Soit $\psi^{*}$ la fonction obtenue à partir de $\psi$ quand on a simplifié $A$ et $\psi$ par les paramètres $\mid x /$. Représentons la valeur moyenne $\bar{\lambda}$ par le symbole

$$
(\varphi) \lambda\left(\psi^{*}\right)
$$

Dans cette symbolique, les exemples présentés au tableau 1 s'écrivent:

$$
\begin{array}{lll}
\left(M^{-1}\right) M(c) & =M_{n} & \text { de Lansing \& Kraemer }{ }^{1} . \\
\left(M^{\alpha}\right) M(c) & =M_{v} & \\
(M) M(c) & =M_{w} & \\
(M) M(c M) & =M_{z} & \\
(D) D\left(c D^{n / 2}\right) & =D_{n+2, n} & \text { de Gralén }{ }^{2} . \\
\left(D^{-1}\right) D(c) & =D_{n} & \text { de Gralén }{ }^{6} . \\
\left(a^{3}\right) a(c) & =a & \text { de Wales }{ }^{3} .
\end{array}
$$

et les différents $s$ moyens calculés de facon formelle au $\S 2$

* Jullander ${ }^{5}$ a introduit des poids moléculaires à deux indices qu'il obtient en introduisant dans la première formule de Svedberg ? les valeurs moyennes dont il a été question jusqu'ici. Ce type de moyenne a été étudié en détails par Singer ${ }^{8}$ mais il sort du cadre de cet article. 


$$
\begin{array}{lll}
\left(s^{-1}\right) s\left(c s M^{-1}\right) & = & s_{n} \\
(s) s(c) & = & s_{w} \\
(s) s(c M) & = & s_{z} \\
\left(s^{-1}\right) s(c) & = & s_{n}^{\prime} \\
(s) s(s c) & = & s_{z}^{\prime}
\end{array}
$$

\section{RESUME}

La formation de valeurs moyennes quelconques est discutée, et un certain nombre de différents types de ces valeurs est présenté, spécialement pour prouver qu'il est possible de former deux différentes catégories de moyennes du type $M_{n}, M_{w}$ et $M_{z}$. Enfin une terminologie nouvelle est proposée.

Nous tenons à remercier ici MM. Gralén et Jullander pour l'aide qu'ils nous ont apportée et les critiques bienveillantes qu'ils nous ont faites au cours du développement de cette étude.

\section{LISTE DES SYMBOLES UTILISÉS}

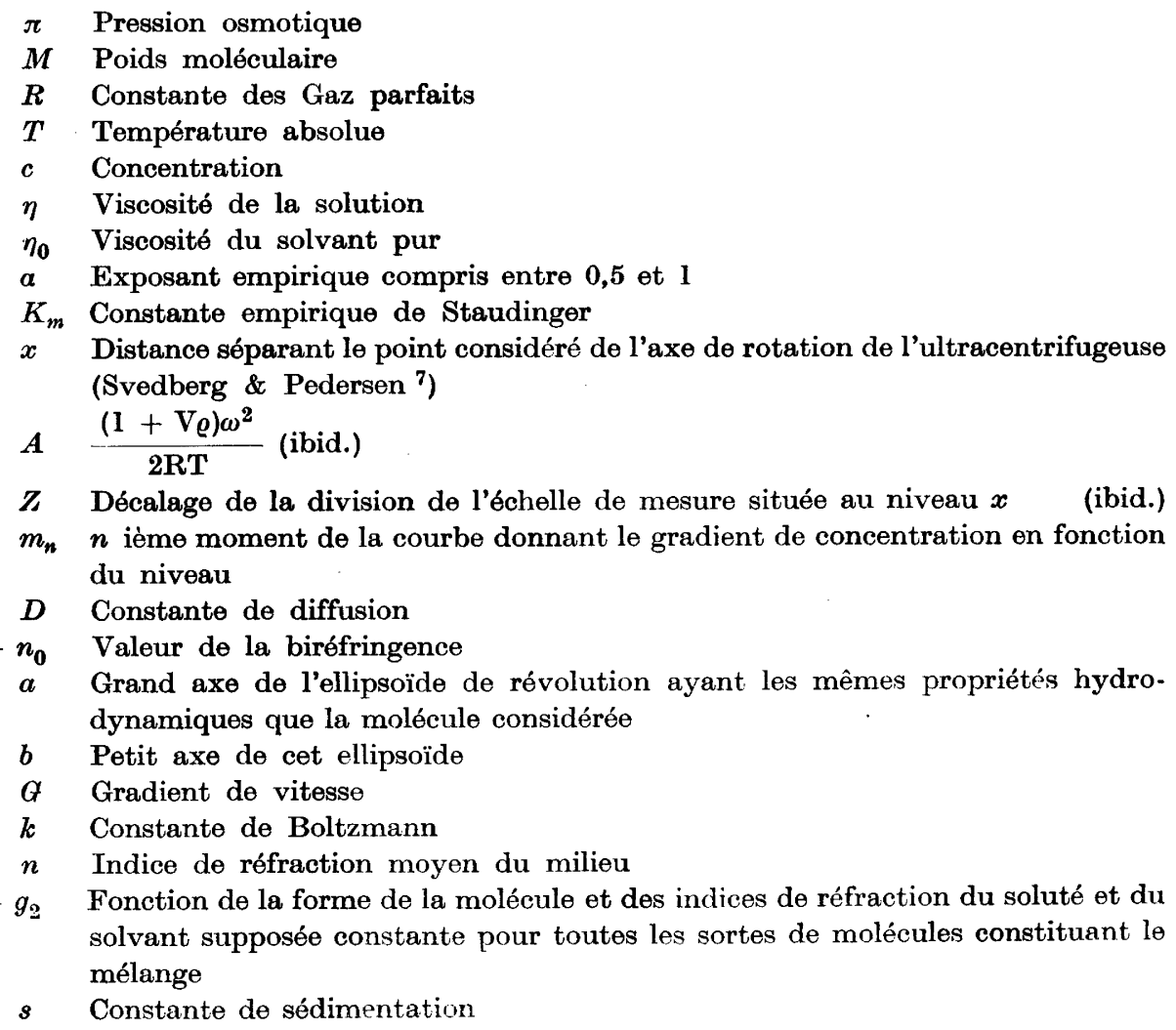




\section{BIBLIOGRAPHIE}

1. Lansing, W. D., et Kraemer, E. O. J. Am. Chem. Soc. 57 (1935) 1367.

2. Gralén, N. Kolloid-Z. 95 (1941) 188.

3. Wales, M. J. Phys. \& Colloid Chem. 52 (1948) 976.

4. Sadron, C. J. phys.radium 9 (1938) 381.

5. Jullander, I. Arkiv Kemi, Mineral. Geol. A 21 (1945) no. 8.

6. Gralén, N. Acta Chem. Scand. 1 (1947) 388.

7. Svedberg, T., et Pedersen, K. O. Die Ultrazentrifuge. Dresden et Leipzig (1940).

8. Singer, S. J. Polymer Sci. 1 (1946) 445.

9. Wales, M. J. Phys. \& Colloid Chem. 52 (1948) 235. 